\title{
ON THE GALACTIC DYNAMO
}

\author{
R. ROSNER and E. DELUCA \\ Enrico Fermi Institute and Dept. of Astronomy and Astrophysics \\ The University of Chicago \\ 5640 South Ellis Avenue \\ Chicago, IL 60687
}

\begin{abstract}
We critically review the status of our current understanding of galactic dynamo theory. In brief, a definitive model for the production of magnetic fields on galactic scales remains to be constructed.
\end{abstract}

\section{Introduction}

The generation of magnetic fields on astrophysical scales remains one of the most challenging problems in astrophysics. Over the past three decades, much progress has been made in overcoming the classical Cowling "anti-dynamo" theorem for axisymmetric systems; and there is now little remaining doubt that large classes of flows exist which have the dynamo property, e.g., which lead to the regeneration of magnetic fields on typical diffusive time scales. In the case of stars and planets, it is absolutely clear that such dynamo action is required, and that appeal to preexisting, primordial fields is in vain: these astrophysical objects show clear evidence for quasi-cyclic magnetic behavior, behavior which is difficult to achieve with primordial fields (Parker 1979).

In the case of galaxies, the requirement to go beyond appeal to primordial fields is not so self-evident. As will be discussed below, the case for requiring galactic dynamos is based on a somewhat subtle point about magnetic field diffusion in galactic disks; and it may still be that primordial fields play a significant role in determining the structure of galactic magnetic fields.

In the following, we propose to briefly review some of the basics of magnetic field generation; to discuss the extant dynamo schemes, first irrespective of the venue of their operation, and then as some of them have been applied to the galactic magnetic field problem; to focus on the basic difficulties these theories face; and finally to discuss what theorists need to know to step beyond current models.

\section{Why Appeal to Dynamos?}

Whenever one discusses magnetic field dynamos, the first question to answer is why go 
to the trouble: Is it in fact necessary to appeal to magnetic field generation during the present epoch, or is it not sufficient to push the problem back to an earlier epoch, e.g., to appeal to primordial fields? In the case of stars, in particular the Sun, the answer is quite straightforward: First, simple comparison of the Ohmic diffusion time scale for fields with spatial scales of order the solar radius with the present solar age shows immediately that even the largest-possible scale primordial field would have by now already decayed significantly. Second, the observed time scale for significant magnetic field variations (e.g., the solar cycle time scale) is far smaller than the Ohmic diffusion time scale. Finally, the observed solar magnetic field is quasi-periodic in time.

Unfortunately, the galactic case is more complex - the above three arguments are irrelevant. That is, a primordial field, if it had been present, could have easily survived diffusion; temporal variations on the diffusion time scale are clearly not observable; and we have no hope of observing periodic behavior. There are only two arguments that we are aware of which do point to the action of a dynamo, neither of which, interestingly enough, is relevant to stars.

The first argument goes as follows (C. Lacey, private communication): Consider the simplest case, namely a primordial field which is uniform on the scale of a forming rotating disk galaxy, and which lies in the rotational plane of this galaxy (except for the special case of a field exactly aligned with the galactic rotation axis, other field orientations lead to a similar conclusion). In that case, if the field is frozen into the protogalactic matter, it will then be "wound up" by the galactic rotation, leading to a toroidal field lying in the galactic plane (this field will have a so-called "bi-symmetric" spiral structure, since there will be at least one field line which passes through the galactic center; Figure 1). Indeed, one can readily show that a steady field structure as shown in Figure 1 is obtained if the field diffusion time scale in the disk is of order the rotational period (as it would be if one adopted an eddy diffusivity; cf. Parker 1979). However, as long as the diffusivity is fairly uniform, the time scale for diffusion in the direction perpendicular to the plane of rotation will be much shorter than the diffusion time in the plane of rotation itself (the ratio of these time scales varies as the square of the ratio of the disk thickness to the galactic radius). This immediately implies that the toroidal field will "leak" out of the galactic disk on time scales much shorter than the time scale on which the bi-symmetric field pattern is established; for this reason alone, we have considerable doubts about an appeal to primordial fields to explain bi-symmetric galactic field configurations.
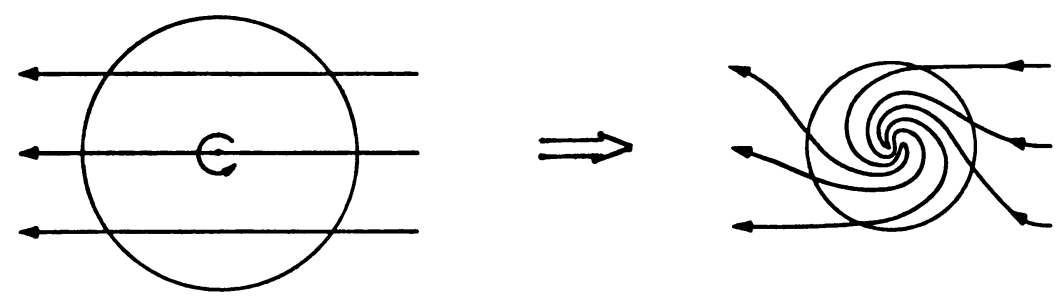

Figure 1: A sketch (after Sofue 1987) of the "bi-symmetric" magnetic field structure which results upon shearing of an initially uniform magnetic field lying in the rotational plane of a disk galaxy by galactic differential rotation (cf. review by Sofue, Fujimoto, and Wielebinski 1986). 
The second argument is equally telling. Unless the Sun is a rather extraordinary star, we have good reasons for believing that of order $5 \times 10^{18} \mathrm{Mx} \mathrm{s}^{-1}$ of magnetic flux are expelled from an average star (cf. Golub, Rosner, Vaiana and Weiss 1981); in fact, it is known that the Sun is a rather inactive main sequence star (cf. Vaiana et al. 1981), so we suspect that this flux loss estimate is a severe underestimate of the actual mean flux loss rate. Assuming of order $10^{10}$ active stars, and a galactic volume comparable to that of our galaxy, one immediately finds that an rms galactic magnetic field $|\delta \vec{B}| \approx 10^{-6} \mathrm{G}$ is built up on a time scale of less than $10^{8}$ years. This field will be characterized by (on galactic scales) very small spatial and temporal integral scales, which are basically set by the local stellar density, the period of the stellar dynamos, and the effective diffusivity on heliospheric scales. However, since each star is a persistent local source of magnetic flux on time scales of several $\times 10^{9}$ years, the actual "life time" of each star-associated magnetic field bubble must be far longer than its temporal integral scale (the latter a measure of the autocorrelation of the local interstellar field associated with a given star). To conclude: the resulting persistent contribution to the galactic field must be highly irregular in both time and space. Since the observed galactic fields do show a degree of order, or coherence, on spatial scales comparable to that of the underlying galaxy, the expelled stellar fields cannot in and of themselves suffice to directly explain the observed interstellar galactic magnetic field, $\langle\vec{B}>$. Now, if there is in addition a large-scale primordial field, $\vec{B}_{o}$, then $|\delta \vec{B}| /\left|B_{0}\right| \gg>1$. Hence, in order to produce the observed largescale field $\langle\vec{B}\rangle$, with $|\langle\vec{B}\rangle| \approx O(|\delta \vec{B}|)$, one requires a dynamo; and any such dynamo model will perforce be very different than current models which presume that magnetic field fluctuations are small when compared to the mean field.

To conclude: There are good reasons for believing that primordial magnetic fields have little to do with presently-observed galactic fields; and one can make a circumstantial case that expelled stellar magnetic fields must be taken into account in any theory for producing the presently-observed fields.

\section{The Essential Ingredients and Limitations of Extant Dynamo Theories}

Figure 2 illustrates the basic elements common to virtually all magnetic dynamo models: In order to regenerate magnetic flux, the fluid motions responsible for dynamo action must (i) stretch and twist flux loops (the $\alpha-\omega$ process; Parker 1979); (ii) reconnect field lines. In a star such as the Sun, internal differential rotation and the Coriolis force acting on rising magnetic flux serve to "stretch and twist"; and turbulent magnetic diffusion is thought to take care of the necessary reconnection. In the case of a galaxy, appeal is usually made to rather similar processes. Whether this works in detail is something we shall return to below; but first, we discuss the various methods used to date in actually quantitatively attacking the dynamo problem.

\subsection{KINEMATIC THEORIES}

The conceptually simplest dynamo models (and, historically, the first ones) are based on the idea that if the magnetic field is sufficiently weak, then one may simply adopt a given flow field, and explore its magnetic field amplification properties without recomputing the back-reaction of the generated fields on the driving fluid motions (Parker 1955). The first successful galactic dynamo models of this sort are due to Parker (1971) and Vainshtein and Ruzmaikin (1971); and in fact virtually all other galactic dynamo models to date have followed in this tradition (cf. Ruzmaikin et al. 1985; Sawa and Fujimoto 1986; Baryshnikova et al. 1987). Thus, one begins with the induction equation 

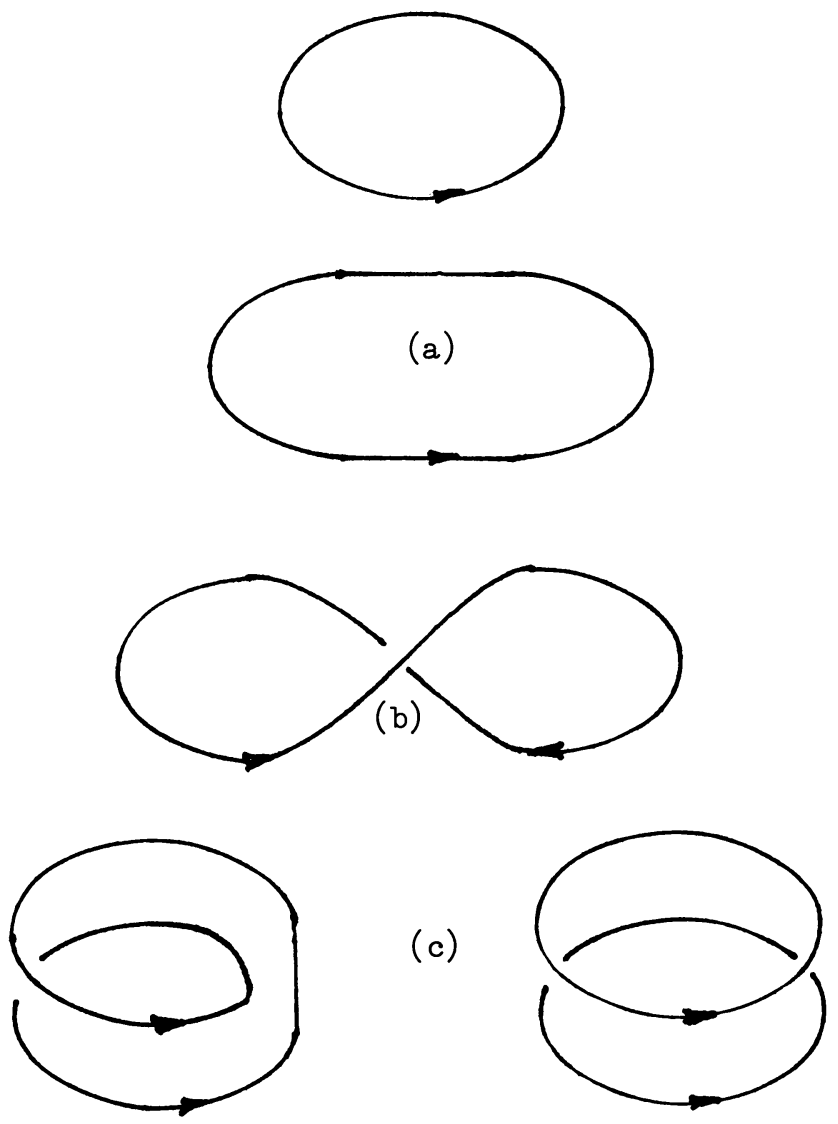

Figure 2: A schematic representation of the principal features of magnetic flux generation (cf. Moffatt 1983): (a) stretching of field lines; (b) half-twist of stretched loop; (c) folding back of twisted loop, and reconnection.

$$
\frac{\partial}{\partial t} \vec{B}=\text { curl } \vec{v} \times \vec{B}+\eta \nabla^{2} \vec{B},
$$

with $\vec{v}$ a specified flow field (that is, a flow whose statistical properties are specified). Upon suitable averaging, one can then obtain an evolution equation for the (ensemble or spatial) mean field, $\langle\vec{B}\rangle$, of the form

$$
\frac{\partial}{\partial t}<\vec{B}>=\text { curl }<\vec{v}>\times<\vec{B}>+\operatorname{curl}(\alpha<\vec{B}>)+\eta_{t} \nabla^{2} \vec{B}
$$

where $\alpha$ is proportional to the mean fluid helicity $\langle\vec{v}$. curl $\vec{v}\rangle, \eta_{t}$ is the turbulent diffusivity, and $\langle\vec{v}\rangle$ is the mean flow velocity. These models have the great advantage that they provide a physically appealing picture for how magnetic flux can in fact be 
produced in a conducting fluid. Furthermore, there is now a considerable understanding of how the mean field equations can be formally derived from the basic equations of motion for a conducting fluid, so that the old concerns about the very possibility of dynamo action - such as Cowling's "anti-dynamo theorem" - can be definitively layed to rest (cf. Moffatt 1978). Nevertheless, virtually no one regards kinematic models as serious representations of, for example, stellar magnetic dynamos; in this case, for which kinematic dynamos were first developed, the observed magnetic fields are strong (so that back-reactions of the fields on the flows cannot be ignored), and turbulent fluid motions are too coherent (the standard mean field dynamo derivations require that the fluid correlation time be short when compared to the typical overturning time scale, whereas typical convective eddies live for times comparable to their overturning time scale). In the case of galactic magnetic fields, the situation is in fact rather similar, so that kinematic models are here equally useful as a didactic tool, but also equally useless in attempts to understand in detail the workings of the actually-observed system. This is particularly true if one recalls that most kinematic theories are inherently linear, that is, they are designed to answer the question: are conditions appropriate for the growth of an unstable magnetic dynamo mode? Thus, they are incapable of representing the fullydeveloped behavior of dynamos.

In order to get around these difficulties, dynamo theorists have pursued two distinct alternatives. In the first, one attempts to model the behavior of a dynamo by clearly defining the important physical processes thought to be operating, and then constructing a set of ordinary differential equations which describe the actions of these processes (cf. Robbins 1976; Cattaneo, Jones, and Weiss 1983; Zel'dovich and Ruzmaikin 1984); the second focusses on the actual numerical simulation of the detailed physics (cf. Gilman 1986; Pouquet 1987; DeLuca and Gilman 1988). It is worth noting that essentially none of these calculations were focussed on the galactic dynamo problem.

\subsection{MODEL DYNAMO EQUATIONS}

As an example of the modeling approach, consider the following set of ordinary differential equations proposed to model the back-reaction of magnetic fields on the differential rotation which largely drives the field amplification (Cattaneo, Jones, and Weiss 1983):

$$
\begin{gathered}
\dot{A}=2 D B-A \\
\dot{B}=i A-\frac{1}{2} i A^{*} \omega-B \\
\dot{\omega}=-i A B-\nu \omega
\end{gathered}
$$

Here $A$ and $B$ represent the poloidal and toroidal magnetic field; $\omega$ is the fluctuating component of the differential rotation; and $\nu$ and $D$ are adjustable parameters. The underlying physics which motivates these equations is straightforward: The first two equations describe a standard $\alpha-\omega$ dynamo, in which differential rotation (measured by $\omega)$ leads to amplification of the toroidal field component, and the Coriolis force leads to conversion of toroidal flux to poloidal flux (note that the diffusivity does not appear explicitly in these equations for the simple reason that units have been adjusted so that time is measured in units of the diffusion time scale). The third equation implements the new physics introduced into the problem - the fact that the poloidal and toroidal fields will both exert forces which tend to reduce the shear, and hence the driving of the dynamo. 
This simple set of equations has the advantage, as just described above, of allowing an economical explanation of the possible types of attainable behavior of fully-developed dynamo solutions, such as the transition from periodic to aperiodic solutions, and the occurence of epochs of complete, but finite duration, quiescence (analogous to the solar Maunder minimum). How useful it is as a tool for understanding galactic dynamos depends heavily on how inspired the insertion of the physics is, something which cannot be decided upon by relying on this approach alone. We shall return to this point of view below.

\subsection{SIMULATIONS}

In principle, numerical simulations avoid the above difficulties entirely: by directly solving the full set of partial differential equations describing the dynamo system, nonlinearities are automatically taken into account, and one expects the important physical processes to emerge naturally as part of the calculation. Unfortunately, things are not as simple as that. For example, P. Gilman and collaborators have, in a series of papers, constructed a more and more realistic model for dynamo action within a star. However, these models remain parametrized; in particular, computer limitations prevent one from modeling the physics over the entire spatial scale range over which important processes are thought to occur. As a consequence, the dynamics on small spatial scales are necessarily subsumed into an eddy diffusivity; this forces a definite commitment to a particular type of sub-grid dynamics, which is for all intents and purposes untestable. One might think that resolving the sub-grid dynamics suffices to resolve this problem. This is however not the case: thus, while calculations such as those of U. Frisch and collaborators (cf. Pouquet 1987) give an accurate picture of how fully-nonlinear dynamo action can take place in a model environment, this model environment is of little interest to astrophysicists (since the effective Reynolds numbers for such simulations are many orders of magnitude smaller than those appropriate to an astrophysical environment.) Thus, we view simulations - whether fully-resolved or not - as tools for allowing us to build physical intuition about the nonlinear dynamo problem, but not as the definitive mechanism for establishing how galactic dynamos really "work".

\section{What are the Problems, and What is Required to Advance Theory?}

Given the inherent limitations of kinematic, linear dynamo theory, and the practical limitations imposed on full simulations by current computer technology, it seems reasonable to suppose that most progress in the foreseeable future will come from modeling. This in turn implies that a premium will be placed on obtaining a clear understanding of the physical processes which must figure in galactic dynamos. For this reason, we focus next on what strike us as the central problems of astrophysical dynamo theory, especially as they pertain to galactic magnetic field production.

\subsection{WHAT IS WRONG WITH "STANDARD" GALACTIC DYNAMOS?}

To begin with, let us consider where we are in the development of dynamo theory, and what the principal remaining problems are. Recall first that virtually all extant galactic dynamo models are kinematic, and hence cannot properly describe the evolution of the $\beta \approx 1$ galactic magnetic field. Buoyancy is typically parametrized, and not explicitly 
taken into account (despite the fact that it is probably the dominant process by which magnetic fields generated in the solar interior and the galactic disk interior reach the outer "surfaces" of these objects.) The fields are assumed to be smooth; that is, there is usually no account taken of the observed filamentation and other fine structure evident in both solar magnetic fields and magnetic fields in our galaxy (although in the galactic case, it is not certain that the structure is in the field rather than in, for example, the particle density distribution). The galactic fluid is viewed as a single-component gas; this means that the effects of neutrals (including such phenomena as ambipolar diffusion) are ignored, contrary to what one would expect (cf. Kulsrud 1987; Zweibel 1988). There is some considerable doubt as to the actual value of the turbulent (eddy) diffusivity; and indeed it has been argued by Ko and Parker (1988) that conditions appropriate to magnetic field generation and turbulent diffusion occur rather sporadically, and so are not the norm. Finally, "standard" dynamo models for thin disks show field symmetries for which there is very little observational support; this is discussed further below.

\subsection{THE SCALE HEIGHT PROBLEM}

A key question for galactic dynamos is what the effective height $h$ of the disk is. The reason is simply that the critical dynamo number (which is a measure of the threshold for marginal stability of the lowest, most-easily excited, mode) scales with the inverse cube of the disk gas scale height. Thus, the threshold for the lowest even mode is (Zeldovich et al. 1983) $|D|>\left|D_{\text {crit }}\right|=8$, whereas the value of $|D|$ in the solar neighborhood is somewhat below this critical value. Since it is commonly agreed that some dynamo action must nevertheless take place, there is substantial motivation to justifying a larger value of $h$, as has been done by Sawa and Fujimoto and collaborators, as well as by Ruzmaikin and collaborators. Alternatively, one could try to argue for (occasionally) enhanced values of the turbulent velocities (cf. Ko and Parker 1988); this also has the effect of suppressing the value of $|D|$ because the dynamo number is a strong function of the rms turbulent velocity.

\subsection{THE MULTI-COMPONENT DYNAMO: IONIZED GAS, NEUTRALS, AND COSMIC RAYS}

As already alluded to, the presence of neutral matter is certain to have a broad impact on magnetic field dynamics in galaxies; this is because the fields, although tied directly only to the ionized particles, are in fact well-coupled to the neutral gas by virtue of neutral-ion interactions. This fact has been recently exploited by Zweibel (1988) in a study of the effects of ambipolar diffusion on standard mean field kinematic dynamos, with the surprising result that one obtains dynamo action even in the absence of any Ohmic or eddy diffusivity; but nonlinear calculations, even of the model type discussed above, are nonexistent. Since the cosmic ray energy density in the interstellar medium is of the same order as that of the relatively low-energy ionized matter, the dynamical effects of cosmic rays on dynamo action also cannot be neglected. As argued by Boehringer and Morfill (1987) and Breitschwerdt, McKenzie, and Voelk (1987), the streaming of cosmic rays out of the galaxy may distend the gaseous galactic halo substantially above the galactic disk. That is, cosmic rays may increase the effective galactic scale height for the ionized gas component, precisely whr.t is required for the standard linear, kinematic dynamo models. 


\subsection{TURBULENT DIFFUSION}

Much has been written about the efficacy of turbulent diffusion of magnetic fields (cf. Knobloch and Rosner 1981), but it remains a challenging task to actually compute the expected magnetic diffusion rate for a given turbulent flow. The difficulty is not so much whether turbulent diffusion acts at all (the observed evolution of solar magnetic fields provides an excellent example for any doubters), but rather the precise prescription for computing the diffusivity. In the case at hand, it is not obvious whether we have sufficient information to even carry this calculation out at all - the naive procedure of simply multiplying a "typical" scale length for interstellar gas motions by a "typical" interstellar gas speed makes implicit assumptions about the nature of random motions in the interstellar medium which are by far not well-established. Indeed, it is not so obvious how turbulent the interstellar medium really is; for example, large-scale, organized flows resulting from the formation of supernovae "bubbles" or "chimneys" (Norman and Ikeuchi 1988) will hardly have the requisite properties for turbulent magnetic field diffusion, and yet occupy a significant fraction of the galactic volume.

\subsection{GALACTIC FIELD SYMMETRIES}

A fundamental property of kinematic dynamos in the thin-disk limit is that the dominant meridional (poloidal) field component is quadrupolar. That is, the linear growth rates of the quadrupolar modes for disks are far larger than those for the dipolar modes, completely the reverse of the usual situation for stars. This is a direct prediction of kinematic theory, and should be directly testable: for example, this predicts that the poloidal field should be mirror-symmetric about the equatorial plane (so that there should be no vertical component to the meridional field at the equatorial plane). There is very little data which can directly shed light on this subject, with the exception of the recent radio and IR observations of the Galactic Center. In particular, the observations of radio arcs by Yusef-Zadeh, Morris, and Chance (1984) which cross the galactic plane, and which show strong evidence from synchrotron polarization of alignment between the arcs and the interstellar magnetic field, seem to provide a direct counterexample to the mirror-symmetric field required by theory. Indeed, Sofue et al. (1986) recognized early on that field configurations of this sort can be obtained by models based on primordial external magnetic fields (and argued for a concentration of fields at the Galactic center, oriented perpendicular to the Galactic plane). Despite this, the arguments presented in $\S$ 2 above regarding the relevance of primordial fields remain in force; hence, one is not left with many possibilities: the only ones that seem to us to be at least somewhat sensible are

(i) that the dynamo region actually extends significantly above the disk (perhaps because of the drag exerted by escaping cosmic rays), so that the thin-disk model becomes irrelevant, and the quadrupolar modes are no longer the dominant modes;

(ii) that the Galactic Center observations are dominated by entirely local phenomena, i.e., that the dynamics governing the large-scale structure of galactic fields is entirely different than that governing the structure of magnetic fields near the Galactic Center.

Only more observations will decide between these two alternatives.

4.6. WHY IS $<|\vec{B}|>\approx B_{\text {equipartition }}$ ?

In stars - or rather, in the Sun - we know that $B^{2} / 4 \pi \approx \rho v^{2}$, but with a very small area filling factor $(f<0.01)$; hence, for stars 


$$
<|\vec{B}|><<B_{\text {equipartition }} \text {. }
$$

In contrast, we know that within the Galaxy $B^{2} / 4 \pi \approx \rho v^{2}$, but with a large areal filling factor $(f \approx O(1))$; hence, interstellar magnetic fields have the property that

$$
<|\vec{B}|>\approx B_{\text {equipartition. }} \text {. }
$$

Why the difference? In the stellar case, turbulent flows are driven by thermal convection. Such flows have a very special planform at the surface: the (cold) downflows are multiply-connected, whereas the (hot) upflows are simply-connected (disjoint). A basic property of such flows, recognized early on by Parker (1963) and Weiss (1966), is that embedded magnetic fields are swept into the boundaries, where they are concentrated to strengths comparable to the flow equipartition value; furthermore, the filling factor of these downflows is well below unity; and indeed recent simulations of $\mathbf{A}$. Nordlund (private communication) show that the concentration of magnetic flux in the strongest downflowing regions lead to quite small magnetic filling factors. In contrast, turbulent flows in the interstellar medium are driven by incoherent sources: supernovae, stellar winds, and so forth. This means that the flows have a complex, multiply-connected topology, and the "sweeping" of magnetic flux into small subvolumes is no longer preferred - there is no longer a mechanism for decreasing the magnetic field filling factor.

\section{Summary}

Our overview of galactic dynamo theory suggests that a convincing explanation for our Galaxy's large-scale magnetic field is not yet at hand. What is clearly required is a more sophisticated understanding of the interstellar medium, and in particular a better appreciation of the roles played by neutrals and cosmic rays; and is it very likely that further major advances will require numerical simulations to a degree not heretofore called for.

Acknowledgments: We would like to express our deep appreciation to R. Hildebrand for our many discussions, in which he disabused us of simple-minded theoretical ideas; and to E.N. Parker for a number of insightful comments on the manuscript. Nevertheless, neither is responsible for any foolishnesses which remain.

\section{References}

Baryshnikava, Y., Ruzmaikin, A.A., Sokoloff, D.D., and Shukurov, A. (1987) "Generation of large-scale Magnetic Fields in Spiral Galaxies", Astron. Ap., 177, 27.

Beck, R. and Graeve, R. (1987) eds., Interstellar Magnetic Fields: Observations and Theory, Springer Verlag, Berlin.

Boehringer, H. and Morfill, G.E. (1987) "On the Dynamical Role of Cosmic Rays in Cooling Flows in Clusters of Galaxies", preprint.

Breitschwerdt, D., McKenzie, J.F., and Voelk, H.J. (1987) "Magnetic Field Structure of the Galaxy and the Formation of Galactic Winds", in Beck \& Graeve (1987), p. 131.

Cattaneo, F., Jones, C.A., and Weiss, N.O. (1983) "Periodic and Aperiodic Behavior in Stellar Dynamos", in Solar and Stellar Magnetic Fields: Origins and Coronal Effects, ed. J.O. Stenflo, D. Reidel Publ., Dordrecht, p. 307.

DeLuca, E.E. and Gilman, P.A. (1988) "Dynamo Theory for the Interface Between 
the Convection Zone and the Radiative Interior of a Star. I. Model Equations and Exact Solutions", Geophys. Ap. Fluid Dyn., 37, 85.

Gilman, P.A. (1986) "The Solar Dynamo: Observations and Theories of Solar Convection, Global Circulation, and Magnetic Fields", in Physics of the Sun, Vol. I, ed. P.A. Sturrock, D. Reidel Publ., Dordrecht.

Golub, L., Rosner, R., Vaiana, G.S., and Weiss, N.O. (1981) "Solar Magnetic Fields: The Generation of Emerging Flux", Ap. J., 243, 309.

Knobloch, E. and Rosner, R. (1981) "On the Spectrum of Turbulent Magnetic Fields", Astrophys. J., 247, 300.

Ko, C.M. and Parker, E.N. (1988) "Intermittent Behavior of Galactic Dynamo Activities", submitted to $A p$. $J$.

Moffatt, H.K. (1978) Magnetic Field Generation in Electrically Conducting Fluids, Cambridge Univ. Press, Cambridge.

Moffatt, H.K. (1983) in Stellar and Planetary Magnetism, ed. A.M. Soward, p. 3, Gordon and Breach Science Publ., New York.

Norman, C.A. and Ikeuchi, S. (1988), submitted to $A p$. J.

Parker, E.N. (1955) "Hydromagnetic Dynamo Models", Ap. J., 122, 293.

Parker, E.N. (1963) "Kinematical Hydromagnetic Theory and its Applications to the Low Solar Photosphere", Ap. J., 138, 552.

Parker, E.N. (1971) "The Generation of Magnetic Fields in Astrophysical Bodies, II. The Galactic Field", $A p$. J., 163, 255.

Parker, E.N. (1979) Cosmical Magnetic Fields, Oxford Univ. Press, Oxford.

Pouquet, A. (1987) "Numerical Simulations of Turbulent Dynamos", in Magnetic Fields and Extragalactic Objects, ed. E. Asseo and D. Gresillon, Editions de Physique, France.

Robbins, K.A. (1976) "A Moment Equation Description of Magnetic Reversals in the Earth", Proc. Nat. Acad. Sci., 73, 4297.

Ruzmaikin, A.A., Sokoloff, D.D., and Shukurov, A.M. (1985) "Magnetic Field Distribution in Spiral Galaxies", Astron. Ap., 148, 335.

Sawa, T. and Fuijimoto, M. (1986) "Bisymmetric Spiral Configuration of Magnetic Fields in Spiral Galaxies. I. Local Theory", Publ. Astron. Soc. Japan, 38, 133.

Sofue, Y. (1987) "Magnetic Fields in Galaxies: The Vertical Fields", in Magnetic Fields and Extragalactic Objects, ed. E. Asseo and D. Gresillon, Editions de Physique, France, 130.

Sofue, Y., Fujimoto, M., and Wielebinski, R. (1986) "Global Structure of Magnetic Fields in Spiral Galaxies", Ann. Rev. Astron. Ap., 24, 459.

Weiss, N.O. (1966) "The Explusion of Magnetic Flux by Eddies", Proc. Roy. Soc. $A, 293,310$.

Vaiana, G.S., et al. (1981) "Results from an Extensive Einstein Stellar Survey", Ap. $J ., 245,163$.

Vainshtein, S.I. and Ruzmaikin, A.A. (1971) Astron. Zh., 48, 902 (English translation Sov. Astron., 15, 714, 1972).

Yusef-Zadeh, F., Morris, M., and Chance, D. (1984) Nature, 310, 557.

Zeldovich, Ya.B. and Ruzmaikin, A.A. (1984) "Dynamo Problems in Astrophysics", Astrophys. Space Phys. Rev., 2333.

Zeldovich, Ya.B., Ruzmaikin, A.A., and Sokoloff, D.D. (1983) Magnetic Fields in Astrophysics, Gordon and Breach Science Publ., New York.

Zweibel, E.G. (1988) "Ambipolar Diffusion Drifts and Dynamos in Turbulent Gases", Astrophys. J., 329, 384. 